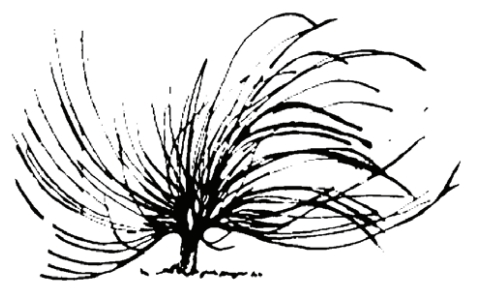

\title{
Estrategias de Enseñanza Innovadoras: un reto para el docente actual
}

\author{
MA. Damaris Cordero Badilla ${ }^{1}$ \\ Universidad Nacional \\ Heredia, Costa Rica \\ jodaro.damaris@gmail.com \\ ML. Ginneth Pizarro Chacón ${ }^{2}$ \\ Universidad Nacional \\ Heredia, Costa Rica \\ gpizarro@una.ac.cr
}

\begin{abstract}
Resumen
El trabajo presenta una visión moderna del proceso de enseñanza que requiere que los docentes se adapten a las nuevas técnicas metodológicas que le permitan estimular, enriquecer y fomentar actividades académicas útiles y efectivas en el proceso de aprendizaje de los estudiantes. Por eso el propósito de este trabajo es analizar aspectos que influyen negativamente en la enseñanza, y sobre estrategias de enseñanza innovadoras que sirvan como herramientas para lograr maximizar el potencial académico de los estudiantes. Primero, se presenta el proceso de enseñanza tradicional y el proceso de enseñanza actual para comprender la necesidad de un cambio significativo en las estrategias metodológicas. Después se presentan los diferentes estilos de aprendizaje y cómo estos se identifican con un tipo específico de estudiante y la forma en que este desarrolla su proceso de aprendizaje. El comprender
\end{abstract}

Recibido: 16 de abril de 2011 - Aprobación: 30 de mayo de 2012

1 Máster en Educación con Énfasis en Enseñanza del Inglés. Investigadora y Académica de la Escuela de Literatura y Ciencias del lenguaje de la UNA, en la Enseñanza del inglés. Ponente en Seminarios Internacionales y Nacionales.

2 Máster en Lingüística. Investigadora y Académica de la Escuela de Literatura y Ciencias del lenguaje de la UNA, en la Enseñanza del inglés. Ponente en Seminarios Nacionales y autora de varios artículos. 
e implementar estos estilos ayudará a visualizar la diversidad en el aprendizaje como parte de cualquier salón de clase. Finalmente, se mencionan diferentes estrategias de enseñanza innovadoras y efectivas que permiten al docente visualizar al educando como un ente activo en el desarrollo de sus habilidades académicas.

Palabras clave: estrategias de enseñanza innovadoras, estilos de aprendizaje, diversidad en el aprendizaje, habilidades académicas

\begin{abstract}
This study investigated the importance of professors to be updated in the use of trendy and innovative teaching strategies. This way, they can implement effective and analytical activities that allow students develop their academic skills. The main purpose of this article is to analyze some aspects that can affect students' learning process negatively, and mention some innovative teaching strategies to maximize their academic skills. First, the traditional and modern teaching methodologies are shown to put into evidence the necessity of a change in the educational system. Second, different learning styles are mentioned to understand the relevance of identifying the student's needs and abilities. Finally, some innovative and effective teaching strategies are suggested to help the professor change his traditional classes and visualize students as active participants in their learning process.
\end{abstract}

Keywords: innovative teaching strategies, learning styles, learning diversity, academic skills

\title{
Introducción
}

\begin{abstract}
Pada vez que un estudiante ingresa al salón de clases, su mayor expectativa es encontrarse con una clase estimulante, creativa e innovadora. Así, el desarrollo de sus capacidades intelectuales será maximizado y aprovechado. De acuerdo con J. Dewey (1933citado por Simpson, 2001, p.197) es tarea del docente generar entornos estimulantes para desarrollar y orientar esta capacidad de actuar de los
\end{abstract}


estudiantes. De este modo, es el maestro quien debe conectar los contenidos del currículum con los intereses y necesidades de los alumnos.

Esta frase explica cómo diversos factores (el desinterés de los alumnos, el aburrimiento crónico y el poco valor que estos le asignan a los contenidos) nos exigen a los docentes pensar y revisar no solo nuestras prácticas y concepciones de la enseñanza, sino también el modo de acercarnos a nuestros alumnos y de guiarlos en el proceso de aprendizaje $y$, fundamentalmente, en su formación como persona.

Así, esta práctica se convierte en un factor esencial que influye y estimula la disposición de dichos estudiantes a aprender y a ser capaces de participar activamente en su propio aprendizaje. Pero, es importante para que esto suceda que el alumno perciba que la construcción de conocimiento debe girar alrededor de sus intereses y su motivación hacia el aprendizaje (Ibarra, 2006).

\section{Antecedentes}

Con la creciente investigación que existe sobre el mejor modo de facilitar el aprendizaje de los estudiantes, hemos aprendido que existen aspectos en cada estudiante que deben ser considerados para que esta tarea se lleve a cabo exitosamente. Aspectos que pueden influir de alguna manera para que estos alumnos pierdan su interés por aprender y que representan grandes desafíos para los profesores.

Basándonos en estas ideas y partiendo de que, "una estrategia de enseñanza equivale a la actuación secuenciada potencialmente consciente del profesional en educación, del proceso de enseñanza en su triple dimensión de saber, saber hacer, y ser" (Sepúlveda y Rajadell, 2001, p. 8), tomamos como objetivo fundamental de este trabajo el investigar sobre aspectos negativos que influyen negativamente en la enseñanza, y sobre la forma en que las estrategias de enseñanza innovadoras pueden ayudar a los educadores a convertir sus salones de clase en lugares de aprendizaje activo para todos sus estudiantes.

\section{Objetivos generales}

- Determinar si el uso de estrategias de enseñanza innovadoras ayudan a mejorar las prácticas docentes para estimular el interés por aprender y el aprendizaje activo de los estudiantes. 
- $\quad$ Proponer un conjunto de estrategias de enseñanza innovadoras que ayuden a mejorar las prácticas docentes con el fin de estimular el interés por aprender y el aprendizaje activo de los estudiantes.

\section{Objetivos específicos}

- Identificar factores psicológicos que puedan afectar la disposición del estudiante a aprender durante la lección.

- Identificar estrategias de enseñanza que puedan afectar la disposición del estudiante a aceptar las estrategias de enseñanza utilizadas por los docentes.

- Analizar estrategias de enseñanza que estimulen el interés por aprender y el aprendizaje activo en los estudiantes.

- $\quad$ Proponer un conjunto de estrategias de enseñanza que ayuden a los profesores a mejorar sus prácticas docentes y a estimular el interés por aprender y el aprendizaje activo de los estudiantes.

\section{Justificación}

En esta época de globalización el docente está obligado a estimular el crecimiento personal y académico de los estudiantes con la idea de que sean capaces de enfrentar el cambiante mundo en el cual se desenvuelven. Por eso, es primordial que todo educador utilice en su salón de clase actividades que atiendan no solo las necesidades personales de los alumnos, sino a sus intereses también. Además, el docente debe replantearse sobre las prácticas de enseñanza que utiliza al abordar los contenidos que enseña, y sobre las mejoras que pueden llevarse a cabo en su uso y aplicación. (Gálvezet al,1999).

Por este motivo, este trabajo tiene como fin el investigar sobre aspectos de cada estudiante que pueden afectar su forma de aprender, así como sobre estrategias de enseñanza innovadoras que podamos utilizar dentro de nuestros salones de clase con la idea de que los estudiantes sean capaces de participar activamente en su propio aprendizaje

\section{Marcoteórico}

La actividad docente que ejecuta todo profesor está inevitablemente unida a los procesos de aprendizaje que realizan los estudiantes, 
y su meta siempre consiste en el logro de determinados objetivos educativos. Por lo que la clave del éxito está en que los estudiantes puedan y quieran realizar las actividades que se planean para ellos, interactuando adecuadamente con los recursos educativos que su profesor les ponga al alcance (Word Press.com, 2008)

Si los estudiantes avanzan positivamente hacia el desarrollo integral de su persona, y de acuerdo con sus capacidades y demás circunstancias individuales, lograrán los aprendizajes planeados para ellos. Por ello el educador debe programar muy bien su actuación ante ellos, y buscar recursos educativos efectivos con el fin de facilitar sus aprendizajes. Esta situación es hecha con la idea de crear entornos de aprendizaje que los motive, oriente y desarrolle habilidades personales e intelectuales. Pero, ¿cómo ha sido el cambio entre el proceso de enseñanza tradicional y el proceso de enseñanza actual?

\section{El proceso de enseñanza tradicional vs el proceso de enseñanza actual}

Anteriormente, la forma tradicional de enseñar era la conferencia o clase magistral en donde el estudiante dependía en gran medida de lo que escuchaba del profesor. Este era quien tenía el conocimiento, y los estudiantes entonces no necesitaban procesar, interpretar o internalizar la información que el educador trataba de transmitirles. El conocimiento era adquirido en forma mecánica; todo se aprendía de memoria y en forma pasiva.

Más recientemente, los procesos de enseñanza han evolucionado hacia otras condiciones en donde se fomenta la interacción entre los estudiantes, la reflexión y la discusión con el objetivo de comprender, que no todos los estudiantes aprenden al mismo ritmo y facilitar un aprendizaje pertinente y duradero. De esta manera, se facilita la disposición del estudiantado a involucrase en las situaciones de aprendizaje (Salas, 2009).

Sin embargo, se hace necesario la búsqueda de prácticas educativas que respondan a los retos actuales que la educación nos presenta, en donde se busca la formación de competencias para la vida en los estudiantes, y se provoca un aprendizaje significativo en ellos. Así, los aprendientes serán capaces de construir su conocimiento personal sobre el mundo que los rodeay lograr que se comprometan con su propio aprendizaje. 
Como parte de este cambio metodológico, el proceso de enseñanza debe relacionarse con lo que el estudiante considere importante o relevante; lo cual le dará la posibilidad de desarrollar competencias para la apropiación de conocimientos (saber qué), habilidades (saber hacer) y valores (saber ser y saber convivir con sus semejantes). Estas competencias les permiten construir conocimientos y el estudiante es el centro de atención; se tomará en cuenta su estilo de aprendizaje y no la materia de estudio recibida por él a través del docente (Salas, 2009).

Existen diferentes estilos de aprendizaje- o distintas maneras en que toda persona aprende- que pueden ayudar a seleccionar las estrategias de enseñanza adecuadas para la actividad docente. Por lo tanto, se procede a continuación a hacer una breve reflexión sobre este tema.

\section{Estilos de aprendizaje}

Durante mucho tiempo los procesos de enseñanza fueron impartidos como si todos los estudiantes tuviesen las mismas formas de aprender y no se contemplaba que cada persona aprende de manera diferente, posee un potencial diferente y tiene conocimientos y experiencias diferentes.

Según la teoría de Kolb (1984, p. 28), los estilos de aprendizaje son "los rasgos cognitivos afectivos y fisiológicos que sirven como indicadores relativamente estables de cómo los alumnos perciben, interactúan y responden a sus ambientes de aprendizaje". Esto define la forma en que cada persona responde y se comporta ante el aprendizaje. Existen cuatro diferentes estilos: alumnos activos, alumnos reflexivos, alumnos teóricos y alumnos pragmáticos. Todo dependerá de si el alumno se implica plenamente en nuevas experiencias, disfruta observando, adapta las observaciones a la teoría o aplica sus ideas en la práctica (Honey y Mumford, 1986).

Cuando se quiere aprender algo, cada individuo utiliza su propio método o conjunto de estrategias. Aunque las estrategias concretas que utiliza varían según lo que quiere aprender, este desarrolla determinadas maneras de asimilar los contenidos, lo cual constituye su estilo de aprendizaje.

Por lo que es común encontrar en el salón de clase grandes diferencias en los conocimientos de nuestros alumnos, a pesar de que han recibido las mismas explicaciones y realizado las mismas actividades y 
ejercicios. Cada uno de ellos aprenderá de manera distinta, tendrá dudas distintas y avanzara más en una área que en otras (Baus, 2010).

Para nosotros los profesores es de gran ayuda tomar en cuenta los diferentes estilos de aprendizaje que existen en nuestros salones de clase, pues esta información nos da la posibilidad de actuar para conseguir un aprendizaje más efectivo y activo en los alumnos, de acuerdo con sus propias características personales (Baus, 2010).

Otra información importante de incluir y explicar en este trabajo es la relacionada con el aprendizaje significativo. Esta teoría de aprendizaje aporta una explicación sobre cómo se aprende. La teoría proporciona un marco de referencia para el desarrollo del proceso educativo, así como para la selección de estrategias de enseñanza que favorezcan este proceso.

\section{Aprendizaje significativo}

En relación con este tema, existe una teoría planteada por $\mathrm{Au}-$ subel en 1973 (citado por Lozano, W. 2008), que plantea que "el factor más importante que influye en el aprendizaje es lo que el alumno ya sabe". Por consiguiente, el aprendizaje de todo estudiante depende de los conocimientos previos que estos pueden relacionar con la nueva información que se les trata de enseñar. Este aspecto es de vital importancia para los profesores ya que les permitirá orientar su labor educativa y aprovechar las experiencias y conocimientos que poseen sus alumnos y que pueden afectar su aprendizaje.

En consecuencia, en el proceso educativo es importante considerar lo que el individuo ya sabe de tal manera que establezca una relación con aquello que debe aprender. Este proceso tiene lugar si el educando tiene en su estructura cognitiva conceptos, ideas y proposiciones estables y definidas para interactuar (Palomino, 2008).

Además, si nuestra meta es promover el aprendizaje activo en nuestros salones de clases, el siguiente tipo de aprendizaje es primordial.

\section{Aprendizaje activo}

El aprendizaje activo es aquel tipo de enseñanza que propone una perspectiva del proceso de enseñanza en el aula basado en el involucramiento de los estudiantes en actividades físicas tales como hablar, escribir, leer, reflexionar, cuestionar, entre otras. Lo anterior, con la idea 
de mejorar su comprensión y retención de los contenidos que se les enseña, así como desarrollar en ellos sus habilidades cognitivas de resolución de problemas y de pensamiento crítico.

Otro de los aspectos importantes de tomar en cuenta también para esta investigación es el interés por aprender que cada uno de los alumnos tenga durante el proceso de aprendizaje, el cual desarrollaremos a continuación.

\section{EI Interés por aprender}

Existen varios factores que afectan normalmente el aprendizaje humano tales como motivación, las emociones, aspectos ergonómicos y ambientales, entre otros que pueden facilitar o dificultar el aprendizaje de nuestros estudiantes, que son importantes de tomar en cuenta a la hora de planear nuestras lecciones diarias. Existen factores tales como la luz, el sonido, la distribución de la clase, la motivación, la responsabilidad, las preferencias sociales que tengan (trabajo individual, grupal, etc.) e incluso preferencias de tipo psicológico, procesamiento de información y de personalidad, que pueden afectar en gran medida su forma de aprender y en el proceso de enseñanza.

\section{Estrategias de aprendizaje}

Brandt (1998, p. 56) define a las estrategias de aprendizaje como" las estrategias metodológicas y técnicas de aprendizaje que varían de acuerdo con los objetivos y contenidos del estudio y aprendizaje de la formación previa de los participantes, posibilidades, capacidades y limitaciones personales de cada quien". En otras palabras, todas aquellas actividades, técnicas y recursos que se planean según los objetivos que se pretende alcanzar y con la finalidad de hacer más efectivo el proceso de aprendizaje. Este representa a su vez componentes fundamentales del proceso de aprendizaje en conjunto con los contenidos, los objetivos y la evaluación de los aprendizajes (Herrera, 2004).

Mientras tanto, aclara Herrera (2004), existe diferencia entre lo que son técnicas de aprendizaje y estrategias de aprendizaje. Las técnicas de aprendizaje son todas aquellas actividades específicas que realizan los estudiantes de forma mecánica cuando aprenden tales como repetición, subrayar, esquemas, preguntas, deducciones e inducciones, 
entre otros. Y las estrategias son guías de acciones conscientes e intencionales que hay que seguir, pues están dirigidas hacia un objetivo relacionado con el aprendizaje.

Además existe otro tipo de estrategias de gran importancia que ayudan a estimular este interés por aprender por parte de los estudiantes, estas son las estrategias de enseñanza.

Según indica Díaz (2003: p.8), las estrategias de enseñanza son todas aquellas actividades que utilizan los docentes en sus salones de clase: los mapas conceptuales, analogías, videos entre otros y las estrategias innovadoras: el aprendizaje centrado en la solución de problemas auténticos, el análisis de casos, el método de proyectos, las prácticas situadas o aprendizaje in situ en escenarios reales, el aprendizaje en el servicio, el trabajo en equipos cooperativos, los ejercicios, demostraciones y simulaciones situadas y el aprendizaje mediado por las nuevas tecnologías de la información y comunicación (TIC) (Díaz, 2003).

\section{Estrategias de enseñanza innovadoras}

El docente planea e implementa diferentes estrategias de enseñanza con el objetivo de transmitir un concepto o una serie de ellas en una forma clara y precisa. Sin embargo, algunas veces no involucra al educando y lo hace ser un participante pasivo del proceso de aprendizaje. Este es un error común entre los profesores ya que estos se visualizan como entes superiores que todo lo saben, cuyo papel en el aula es trasmitir todo su conocimiento sin que exista una retroalimentación por parte de los estudiantes. Definitivamente, esta metodología de enseñanza está desapareciendo de las aulas y una nueva metodología ha emergido como una forma de potenciar la capacidad intelectual y personal de los educandos.

Por lo tanto, la necesidad de estrategias de enseñanza innovadora e interactiva será sugerida como una alternativa para lograr un cambio de la enseñanza tradicional en las aulas a una que potencialice al estudiante a lo largo de su proceso de aprendizaje. A continuación se mencionarán algunas:

1. Entornos estimulantes. El salón de clase debe ser concebido como un lugar armonioso donde el aprendiente tenga las condiciones adecuadas para escuchar, interiorizar y desarrollar lo 
trasmitido por el docente. Esta situación se logra si dentro del planeamiento del curso, el profesor incluye actividades introductorias donde simplemente se discutan temas relacionados con la temática del día, pero que no requiera que el estudiante haya leído sobre este. Así, su conocimiento general sobre el tema lo hará sentirse un poco más seguro de lo que se discutirá, en una forma más específica, durante el resto de la clase. Un entorno tranquilo y alegre estimula una actitud positiva y receptiva por parte de los estudiantes.

2. La relación de los contenidos del currículo y las necesidades de los estudiantes. Los contenidos de un curso y la forma clara como se presentan son fundamentales para que este se desarrolle exitosamente. Por lo tanto, estos contenidos deben responder a las necesidades de los estudiantes y deben adaptarse a estas. Cada contenido debe tener un objetivo específico para desarrollar una habilidad, corregir un problema, incrementar el conocimiento sobre un tema determinado y expandir el uso de una forma o conocimiento en otras áreas, lo cual se puede lograr por medio del análisis de los objetivos del curso y como estos serán herramientas para que el estudiante adquiera habilidades ya sea para el aprendizaje de una lengua, el desarrollo de habilidades matemáticas, musicales, deportivas u otras.

3. Aburrimiento crónico. Frecuentemente los estudiantes tienden a referirse a una clase como aburrida y poco estimulante. Dicha situación conlleva a que muchos no asistan a clases, deserten o simplemente lleguen a recostarse sobre sus mesas, mirar a través de la ventana o se abstraen sin prestar atención a la clase. Una forma de remediar este problema es el uso de actividades que combine la teoría y la práctica. Durante la primera parte de la clase, los estudiantes están más ansiosos por recibir información y prestar atención detallada. En este momento, el docente debe introducir los aspectos teóricos en una forma clara y precisa. Es conveniente no leer continuamente; por el contrario, la explicación en una forma de diálogo estimulará la atención de los estudiantes y su deseo de interactuar con el docente. Durante la segunda parte de la sesión, actividades que requieran el uso práctico de los conocimientos adquiridos previamente serán fundamentales para que los educandos despierten la capacidad analítica de los estudiantes y estos contextualicen la teoría. La 
teoría debe ser percibida como una base para poder desarrollar habilidades y actividades en la práctica cotidiana.

4. El autoanálisis. El mayor problema de un estudiante es su incapacidad de autoanalizarse y darse cuenta de cuáles son las debilidades que están afectando su proceso de aprendizaje. Esto hace que el aprendiente no pueda desarrollar estrategias remediales y cumpla con las expectativas del curso y las propias. Una forma de lograr el desarrollo del autoanálisis es por medio de actividades que lo obliguen a cuestionar lo discutido en clase, con el fin de monitorear su capacidad de análisis y discusión sobre los temas del curso. Además, la asignación de tareas que enfaticen un monitoreo constante de su capacidad de comprensión, investigación y solución de problemas lograran que desarrolle estrategias de aprendizaje que le servirán para cualquier curso.

5. Creatividad. Ser creativo implica ir más allá de lo enseñado y aprendido en el aula. El docente debe planear actividades interactivas que sean un reto para el estudiante y estimulen su deseo de aprender y cuestionar lo dicho. Existen diversas formas de fomentar la creatividad en los aprendientes:

a. El uso de proyectos cortos que investiguen aspectos poco discutidos en la clase o que ayuden a comprender conceptos o aspectos relevantes sobre el tema en cuestión.

b. El uso de foros, chats, blogs u otra forma de interacción que estimule el compartir puntos de vista y conocimientos entre los estudiantes y el docente.

c. El uso de actividades dentro de la clase que desarrollen el interés sobre temas adyacentes que no están incluidos dentro de la temática del curso.

6. Expectativas sobre el curso. Todo curso contempla una serie de expectativas por parte del docente. Este debe tener claridad sobre el perfil de salida de los estudiantes y lo que deben desarrollar durante el curso. Por lo tanto, cada una de las actividades planeadas y desarrolladas deben ser guiadas a cumplir estas expectativas. El cumplimiento de estas será influenciado por el enfoque del docente hacia la temática y la metodología aplicada. Un enfoque claro y preciso logrará que los educandos alcancen el perfil de salida requerido de acuerdo con el nivel de competencia y logros requeridos. 
7. Aceptación del error. El docente frecuentemente visualiza el error como un proceso que debe ser castigado y anulado como parte del proceso de aprendizaje. Sin embargo, el error puede ser aceptado como un proceso de mejoramiento que estimule al estudiante. Cada vez que un estudiante comete un error ya sea en un examen, trabajo, exposición oral o análisis de un problema es amonestado y estimulado a no cometerlo bajo pena de castigo. Así, el aprendiente evita a toda costa el cometer errores y lucha por alcanzar la perfección. Este hecho debe cambiar radicalmente; el error es necesario en el proceso de aprendizaje ya que estimula el autoanálisis y el deseo de mejorar las debilidades y afianzar las fortalezas. Un estudiante que comprenda que el error en la clase no es castigable, sino merecedor de discusión para su mejoramiento aprenderá a luchar por ser más analítico consigo mismo y los demás.

8. El conocimiento- un estado permanente. La trasmisión de conocimiento no debe ser vista como un proceso transitorio para poder realizar un examen, proyecto o tarea asignada. El docente debe fomentar entre sus estudiantes que lo aprendido en la clase será una herramienta útil para el desarrollo de las diferentes actividades que desarrollarán en su vida. Este conocimiento será permanente y provechoso y su uso en actividades académicas y cotidianas fomentará seres humanos capaces de resolver problemas, plantear discusiones y trasmitir ideas fundamentadas.

9. Retos. Una clase que no fomente los retos y estimule las aspiraciones de mejoramiento académico no está cumpliendo un principio básico de la educación: crear seres pensantes y autónomos intelectualmente. El llevar a los estudiantes a la orilla para obtener lo mejor de ellos hace que estos desarrollen habilidades inimaginables. Cada reto conlleva un objetivo dentro del curso; el estudiante aprenderá a exigir lo mejor de sí y el docente probará que el demandar excelencia académica brindará beneficios al estudiante y a su proceso de aprendizaje.

10. Adaptabilidad de las estrategias de enseñanza. Creer que todos los estudiantes son iguales y aprenden bajo las mismas condiciones es una idea totalmente alejada de la realidad. Actualmente, los diferentes tipos de estudiantes que se pueden encontrar en una clase requieren que el docente visualice, planee y adapte sus 
estrategias de enseñanza. Así, cada una de las actividades planteadas de acuerdo con un objetivo específico requerirá que se utilicen diferentes metodologías que satisfagan las necesidades de cada estudiante. Es imposible enseñar una temática de acuerdo con cada uno de los estudiantes; sin embargo, es posible que la distribución de cada actividad sea dirigida a un grupo en particular. Al final del curso, todos los aprendientes serán expuestos a alguna actividad que satisfaga sus necesidades académicas y las estrategias metodológicas serán variadas y efectivas.

\section{Conclusión}

El proceso de enseñanza requiere algo más que una pizarra y un marcador en un salón de clase. El docente actual debe estar capacitado para implementar estrategias de enseñanza que le permitan lograr una efectiva realimentación en el aula lo cual permitirá que el estudiante perciba el salón de clase como un entorno agradable, amigable y placentero. Existen varios aspectos que deben ser tomados en cuenta cuando se planea una sesión académica efectiva. Primero, el docente debe comprender que la clase está conformada por una gran diversidad de estudiantes. Cada uno tiene deseos de aprender, recibir elogios por sus logros académicos, desarrollar sus habilidades y potenciar sus fortalezas. Así mismo, estos estudiantes experimentan el temor al fracaso, la ansiedad y la frustración. Por lo tanto, es deber fundamental del docente adaptar su estrategias de enseñanza a cada uno de sus miembros, fomentando la inclusión y el desarrollo académico.

Segundo, la clase debe fomentar el interés por adquirir conocimiento en una forma permanente y acumulativa. Solo de esta forma, el estudiante podrá afrontar los retos académicos que se le presenten en otros cursos o en su desempeño profesional. Así, las estrategias de aprendizaje estarán enfocadas a fortalecer la comprensión, el análisis y la discusión del tema en estudio. Tercero, el uso de estrategias de enseñanza innovadoras enriquece la sesión y despierta el interés de los estudiantes por investigar, analizar y divulgar lo aprendido. Los recursos tecnológicos como los foros, blogs, chats y skipe facilitan el desarrollo social y la interacción profesor- alumno y alumno- alumno. Definitivamente, la innovación es imprescindible para que un docente desarrolle habilidades en la enseñanza y enriquezca el aprendizaje de 
sus estudiantes. Por lo tanto, cada uno de estos factores influirá en el éxito de una sesión académica efectiva, cuya finalidad primordial será el buen desempeño académico de los estudiantes.

La implementación de estrategias de enseñanza innovadoras y que incluyan los aspectos antes mencionados no siempre son fácilmente aceptadas. Algunas veces son rechazadas o juzgadas negativamente por los docentes, los cuales tienden a visualizar el cambio de una enseñanza tradicional a una más innovadora como un factor negativo. El temor al cambio se incrementa cuando los docentes deben actualizar las estrategias de enseñanza e incursionar en nuevas estrategias que requieren estudio y planeamiento detallado. Sin embargo, esto no debe ser visto como algo extraño o experimental. El cambio en las estrategias metodológicas, el entorno y la forma de visualizar al educando son aspectos positivos que traerán beneficios adicionales al desarrollo de la clase y al desarrollo académico del estudiante.

El estudiante actual se desarrolla en un mundo cambiante que demanda una actualización constante no solo en el área tecnológica, sino en el área intelectual. El saber es parte integral de su vida y el docente es parte fundamental de su aprendizaje. El docente es un ente facilitador de conocimiento que permite que los educandos se adapten a la clase, a su entorno y posteriormente a su lugar de trabajo. Así, el estudiante es un participante activo y deseoso de desarrollar su potencial académico y humano. Finalmente, los diferentes estilos de aprendizaje permiten enriquecer el proceso de aprendizaje de los estudiantes y su conocimiento, y el docente es el instrumento que guía y fortalece ese proceso utilizando estrategias específicas para desarrollar los objetivos trazados en la clase.

\section{Referencias bibliográficas}

Baus, T. (2010). Los Estilos de Aprendizaje. Recuperado de URL: http:/www.monografias.com/trabajos12/losestils/losestils.shtml

Díaz Barriga, F. (2003). Cognición situada y estrategias para el aprendizaje significativo .Revista Electrónica de Investigación Educativa, 5 (2).

Gálvez, J. et al. (1999). Interés por Aprender Vs. Necesidad de Aprobar. Argentina. Córdoba: Universidad de Córdoba - Facultad de Psicopedagogía.

Herrera, Gabriel (2004), Estrategias de Aprendizaje. Recuperado deURL:http:/www.monografias.com/trabajos19/estrategias-aprendizaje/estrategias-aprendizaje.shtml 
Ibarra, L. (2006). El Role del Profesor. Universidad de La Habana. Facultad de Psicología. Recuperado de URL: http://www.psicologia-online.com/articulos/2006/ rol_profesor.shtml

Palomino, W. (2008). Teorías de Aprendizaje Significativo de David Ausubel. Recuperado de URL: http://www.monografias.com/trabajos6/apsi/apsi.shtml

Salas, M. (2009)Del Proceso de Enseñanza Aprendizaje Tradicional, al Proceso de Enseñanza Aprendizaje para la Formación de Competencias, en los Estudiantes de la Enseñanza Básica, Media Superior y Superior. La Habana: Ministerio de Ciencia, Tecnología y Medio Ambiente.

Sepulveda, F. y Rajadell, N. (2001). Los procesos formativos en el aula: Estrategias de enseñanza y aprendizaje. Recuperado de URL: http://www.upm.es/innovacion/ cd/02_formacion/talleres/nuevas_met_eva/rajadellarticulo.pdf

Simpson, D. (2001). John Dewey's Concept of the Student. Recuperado de: http://www. csse.ca/CJE/Articles/FullText/CJE26-2/CJE26-2-Simpson.pdf

Lozano, W. (2008). La Estructura Cognitiva Como Base Del Trabajo De Ausubel. Recuperado de:www.articulosinformativos.com/La_Estructura_Cognitiva como_Base_del_Trabajo-a1156178.htm

WordPress.com. (Marzo, 2008). Aprender a enseñar teniendo en cuenta la diversidad. Recuperado de: portal.perueduca.edu.pe/basicaespecial/articulos/art01_01-0906.pdf 